\title{
The sine-Gordon equation in reversed-field pinch experiments
}

\author{
J. L. Shohet, ${ }^{\text {a) }}$ B. R. Barmish, and H. K. Ebraheem \\ Department of Electrical and Computer Engineering, University of Wisconsin-Madison, Madison, \\ Wisconsin 53706
}

A. C. Scott

Department of Mathematics, University of Arizona, Tucson, Arizona 85721

(Received 6 January 2004; accepted 28 April 2004; published online 8 July 2004)

\begin{abstract}
The focal point of this paper is a nonlinear model which describes localized magnetohydrodynamic modes in reversed-field pinch experiments. To date, nearly all experimental and theoretical work in this area have relied on the use of Fourier decomposition of spatial variations as a function of time. Moreover, due to the complexity of this nonlinear problem, previous work is restricted to the analysis of a relatively small number of modes. In contrast, the model studied in this paper, based on the sine-Gordon equation, addresses the full nonlinearity, does not rely on Fourier decomposition and does not require the range of the nonlinearity to be small. A specific consequence of working with the full nonlinearity is the existence of solitary waves in dispersive media. These solitary waves, a key part of the model, are used to describe the so-called slinky-mode propagating in the plasma. To this end, a remarkable resemblance is seen between the wave forms obtained from experiments and the mathematical predictions of the model. (C) 2004 American Institute of Physics.
\end{abstract}

[DOI: $10.1063 / 1.1763914]$

\section{INTRODUCTION}

The focal point of this paper is a nonlinear model ${ }^{1}$ which describes localized magnetohydrodynamic (MHD) modes in reversed-field pinch (RFP) experiments. To date, nearly all experimental and theoretical work involving these so-called slinky modes ${ }^{2}$ has relied on the use of spatial Fourier decomposition as a function of time. Moreover, due to the complexity of this nonlinear problem, previous work has been restricted to the analysis of a relatively small number of Fourier components. ${ }^{3}$ In contrast, the model studied in this paper, based on the sine-Gordon equation, addresses the full nonlinearity, does not rely on Fourier decomposition, and does not require the range of the nonlinearity to be small. A specific consequence of working with the full nonlinearity is the existence of solitary waves in dispersive media. These solitary waves, a key part of the proposed model, are used to describe the slinky mode propagating in the plasma. In this regard, the motivation for this work is derived from the fact that the use of linearized models in a dispersive medium does not result in such solitary waves that sustain themselves as they propagate. Support for the model is provided by data obtained from the Madison symmetric torus (MST) reversedfield pinch experiment operated at the University of Wisconsin-Madison. To this end, a remarkable resemblance is seen between the wave forms obtained from experiments and the mathematical predictions of the model.

\section{A. Previous work}

Theoretical $^{4-11}$ and experimental ${ }^{2,12-21}$ work involving mode locking, magnetic field errors, and rotational control through resonant magnetic perturbations has been performed

${ }^{a)}$ Electronic mail: shohet@engr.wisc.edu on several RFP and tokamak devices; among the RFP experiments are the MST, ${ }^{2,14,21,22}$ the reversed field experiment $(\mathrm{RFX}),{ }^{19}$ and the toroidal pinch experiment-RX (TPE-RX). ${ }^{15-17,23}$ In MST, the dynamo modes, which are responsible for generating the reversed magnetic field, continually exhibit a sawtooth behavior as a function of time ${ }^{2}$ as shown in Fig. 1. In many cases, the sawtooth-mode amplitude gradually increases from small values until a critical value is reached at which a rapid magnetic reconnection event, termed a sawtooth crash, is triggered. After the crash, the mode amplitudes return to their initial values, and the process may repeat itself.

It should be noted that the dynamo action that maintains the field reversal is only significant during sawtooth crashes, and this is due to the large amplitude of the fluctuations. ${ }^{4,5}$ This intermittency of the MHD dynamo action is a common feature of RFPs. ${ }^{4,5}$ This paper is concerned with the time intervals between successive sawtooth crashes where the slinky-mode dynamics may be carefully studied.

\section{B. The slinky mode}

It has been observed that during the sawtooth cycles, well before a crash, the fluctuating MHD modes in a RFP may become locked in phase to form a "slinky mode" which is a toroidally localized, rotating magnetic perturbation. ${ }^{22,24}$ This then gives rise to a toroidally localized, rotating "hot spot" induced on the surface of the conducting shell that faces the plasma. Slinky modes have at times been observed to lock to static error fields, and therefore stop rotating in the laboratory frame. ${ }^{22,24}$ Unfortunately, when this occurs, the associated "hot spot" also stops rotating and rapidly overheats the plasma-facing chamber surface at this point, leading to an influx of impurities into the plasma, and the even- 


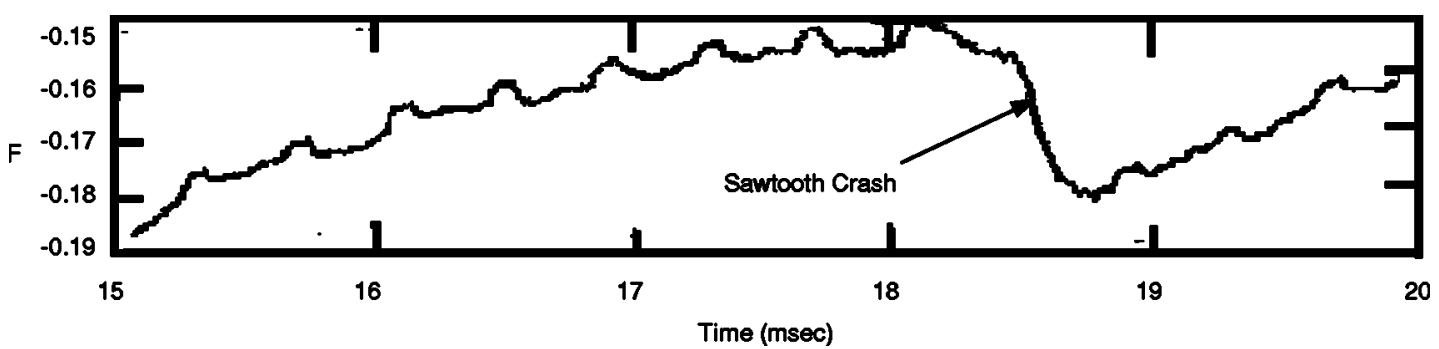

FIG. 1. Illustration of a Sawtooth Crash. The quantity $\mathrm{F}$ is a measure of the level of field reversal. The more negative $\mathrm{F}$ is, the lower the energy of the configuration.

tual termination of the discharge. The plasma current in the RFX (Ref. 4) experiment, for example, has been shown to be limited as a direct consequence of the problems associated with locked slinky modes.

Most notably, the dominant mode-coupling mechanism in RFPs, being a consequence of nonlinear interaction, leads to a number of problems involving different Fourierdecomposed MHD modes inside the plasma. ${ }^{6}$ Since this kind of coupling is difficult to analyze, in the past, in order to make any progress, previous authors have limited the number of Fourier-decomposed modes that are taken into account. In the tokamak, which also exhibits MHD mode locking, ${ }^{10}$ a set of nonlinear equations is used to describe the amplitude and frequency of Fourier-decomposed tearing modes and their related phase instabilities. This is based on experimental measurements of amplitude- and phase-tuned oscillating currents linked to the measured signals by a feedback loop. ${ }^{10}$

In most fusion plasmas, nonlinear interactions are central to the determination of plasma behavior. In particular, we argue that the well-known slinky mode itself is a manifestation of a fully nonlinear phenomenon. We now describe the way in which Fourier decomposition has been used to analyze what are likely to be nonlinear phenomena in reversedfield pinches.

In MST, three-wave nonlinear coupling of Fourierdecomposed magnetic fluctuations in momentum space $(k$ space) was observed. ${ }^{21}$ It was found that during the sawtooth-crash phase, the nonlinear coupling is strongly enhanced, with a simultaneous broadening of the mode spectra. $^{21}$ Phase-locked modes were almost always observed to lock to the wall in close proximity to the poloidal gap. (Reversed-field pinch devices have a characteristic set of insulating intersecting gaps cutting the vacuum chamber in the toroidal and poloidal directions.) In another RFP experiment TPE-RX, ${ }^{16}$ it has been suggested that the locking phenomenon may be due ${ }^{16}$ to the braking effect of eddy currents induced in the vacuum vessel by the growing, phase-locked modes. In addition, it was also proposed that locking is enhanced by the presence of a "halo" current, which is the current that flows through the plasma "scrape-off" layer into the vacuum chamber at the locking location. ${ }^{17,23}$

Experiments that have been carried out on MST (Ref. 14) show that an externally applied magnetic perturbation, produced by a set of eight coils at the toroidal gap, causes locking of the dominant Fourier-decomposed magnetic modes for a poloidal mode number $m=1$, and toroidal mode numbers $n=5$ through 10 , when the perturbation is made resonant with the Fourier-decomposed magnetic modes. A perturbation which is not in poloidal resonance (for example, $m=0$ or 2) produces no locking.

In the RFX reversed-field pinch, ${ }^{19}$ experiments were performed that involved producing an external rotating magnetic perturbation and locking it to an internal rotating Fourier-decomposed MHD mode. It was found that in addition to changing the rotation of the targeted Fourierdecomposed mode, modes of different helicities could be made to rotate in such a way as to satisfy a three-wave nonlinear resonance condition. ${ }^{21}$

\section{Consistency with the sine-Gordon model}

A main objective of this paper is to demonstrate that our model, ${ }^{1}$ based on the sine-Gordon equation, produces predictions which are consistent with experimental observations. For example, when we consider the analytical investigation of mode locking, ${ }^{4}$ which involves damping due to electromagnetic torques acting in the vicinity of rational surfaces, we include the effects of the helical eddy currents and other damping by incorporating appropriate damping terms in the model equation. Both the MST and RFX experiments are constructed with conducting shells, but RFX also has an inner toroidal resistive (lower-conductance) shell. There are observed experimental differences in the rotation of dynamo modes in MST and RFX. It was found that the vacuumvessel eddy currents in the resistive shell are the primary cause of the observed lack of dynamo-mode rotation in RFX. The corresponding eddy currents in MST were found to be too weak to cause a similar problem. ${ }^{4}$ Theoretical models for slinky mode locking have been developed ${ }^{8,13}$ in the presence of a resistive vacuum vessel with a locking torque due to a resonant error field. These models consist of a set of timephase evolution equations which describe the behavior of the slinky mode in RFP under the influence of the braking torque due to eddy currents which are excited in a resistive or perfectly conducting ${ }^{11}$ vacuum vessel. These models were developed with the utilization of a Fourier decomposition of the slinky mode.

In the case of a perfectly conducting vacuum vessel, which applies to the MST experiment, one analysis 
concludes ${ }^{11}$ that phase locking appears when the magnetic energy of the Fourier-decomposed modes $m=1, n=5$ and $m=1, n=4$ is maximized and that the phase locking continues as long as the $m=1, n=5$ mode remains dominant. A conclusion to be drawn from such analyses is that phase locking of these modes is the cause of the localized slinky mode formation. However, an alternate conclusion is that when the slinky mode is formed, phase locking takes place as a "marker" for the presence of the slinky mode. That is, any disturbance which is localized in space will, as a consequence, exhibit phase locking. We address this issue later on in this work.

To summarize, nearly all of the experimental and theoretical work in this area has relied on the use of Fourier decomposition of spatial variations as a function of time. Nonlinear interactions between the Fourier-decomposed modes are assumed, but due to the complexity of the problem, only a relatively small number of such modes are analyzed. For example, in Ref. 5 it is stated as follows.

"The dominant mode coupling mechanism in RFPs is that due to the nonlinear interaction of different Fourier decomposed MHD modes inside the plasma. Unfortunately, this type of coupling is far more difficult to analyze than the toroidal coupling that takes place in tokamaks. In order to make any progress, it is necessary to severely limit the number of Fourier decomposed modes that are taken into account during the analysis."

The limitations above are necessitated by the fact that sinusoids are not eigenfunctions of a nonlinear equation. As a result, attempting to use sinusoids in such a representation to find a solution to the nonlinear equation introduces additional mode creation and coupling between all of the components, resulting in a significant increase in the complexity of the problem.

Similar to theory, in experiments, spatial Fourier decomposition is often performed as a function of time $^{3}$ and the evolution of the mode amplitudes and phases are then analyzed in order to predict the behavior of the system as a function of time.

\section{Limitations of Fourier decomposition}

In view of the discussion above, we build upon existing results for classes of systems for which linearization arguably does not apply. To this end, we introduce a technique that does not require mode analysis and thus is not limited to the interaction of a small number of modes. We view this as a critical point not only because our analysis does not require Fourier decomposition, but also because modal analysis for nonlinear systems is only valid for time and space restrictions which limit the range of nonlinearity to be small $^{25}$ and eliminates the emergence of fully nonlinear phenomena. Said another way, such models, based on a linearizing assumption or a restriction of the number of modes, imply that a linear or small combination of mode amplitudes will adequately represent the solution to a nonlinear equation. The nonlinear model studied here does not rely on this assumption, and as a result, significant differences in the solutions can emerge.
With existing literature in mind, after development of the model, we compare our theoretical predictions with experimental measurements by carrying out a time-varying Fourier spatial decomposition.

\section{E. Nonlinearity in dispersive media}

For solitary waves in dispersive media, use of linear theory leads to periodic propagating disturbances in many systems, corresponding to the elementary solutions $\exp (i k x$ $-i \omega t)$. For waves of moderately small amplitude in what might be called "near-linear" or "quasi-harmonic" theory, results are obtained by perturbation methods based on small amplitude expansions and a Fourier analysis description. In the full nonlinear theory, however, while the solutions are no longer sinusoidal, the existence of periodic solutions in the traveling wave variable $\zeta=k x-\omega t$ can nevertheless be established in a number of cases. The main nonlinear effect is not simply the difference in functional form; it is the appearance of amplitude dependence in the dispersion relation. This leads to new qualitative behaviors, which are not merely a correction of linear formulas. For such nonlinear forms, superposition of solutions (mode summation) is no longer available to generate a complete solution.

A specific consequence of our nonlinear model is the existence of solitary waves in dispersive media. While waves with these profiles disperse in the linear theory, the inclusion of nonlinear effects counterbalances the dispersion to produce waves of permanent shape; e.g., the slinky mode in reversed-field pinches. Much previous theoretical work for the slinky mode is based on plasma dynamics in cylindrical geometry. Since the slinky mode is a spatially localized phenomenon and the plasma is dispersive, nonlinearities need to be introduced so as to cancel out the dispersive effect. It is our hypothesis that by implementing the full nonlinear theory in reversed-field pinches, including the effects of toriodicity, and recognizing the fact that magnetized plasmas support helical waves, the sine-Gordon equation naturally emerges. The sine-Gordon equation has an inherent balance between nonlinearity and dispersion leading to solitary-wave kink structures that emerge from highly nonlinear dynamics in dispersive media but does not require the introduction of any other nonlinearities.

\section{EXPERIMENTAL CONFIGURATION AND THE NONLINEAR MODEL}

This section reviews our nonlinear model ${ }^{1}$ which we propose as a description of the motion of the slinky mode that has been observed to travel in a helical path around the reversed-field pinch experiment at the University of Wisconsin-Madison, MST. In previous work, ${ }^{1}$ this model was introduced to study the conditions for locking of the MHD mode based on experimental results and the probability of a discharge locking as a function of key variables. In contrast to our previous results, this work solves the key nonlinear partial differential equation numerically rather than with a perturbation method. Use of the numerical technique allows details of the shape and dynamics of the slinky mode to be readily apparent, conveniently examines the dynamics of multiple kinks, and permits spatial Fourier decomposition. 


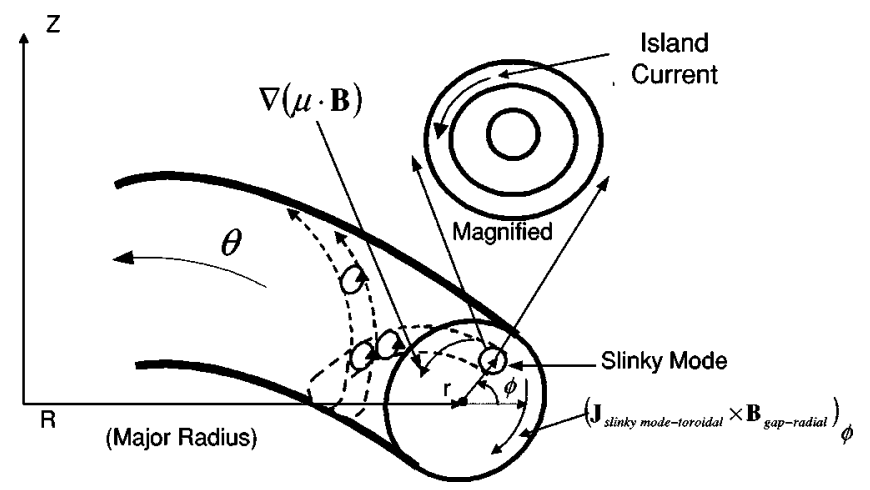

FIG. 2. A conceptual drawing of the slinky mode in the MST torus. The mode threads its way along the torus passing both the poloidal and toroidal vacuum chamber gaps.

The latter, which is a key objective of this work, provides a means to consider this model vis-a-vis the existing literature on Fourier decomposition.

\section{A. Experimental configuration}

The MST experiment is a large reversed-field pinch with a minor radius of $0.52 \mathrm{~m}$ and a major radius of $1.5 \mathrm{~m}$. The aluminum toroidal vacuum chamber wall is $0.05 \mathrm{~m}$ thick except for two insulating gaps-one that cuts the vacuum chamber toroidally and one that cuts the chamber poloidally. Depending upon the values of various parameters, the probability of locking (the mode becomes stationary and causes the discharge to expel its energy) changes. A set of $32 \mathrm{mag}-$ netic fluctuation pickup coils are spaced uniformly around the torus toroidally at a fixed poloidal angle that is used to detect the presence and dynamics of the slinky mode.

\section{B. Slinky mode and kink soliton}

Based on the observed properties of the slinky mode along with an analysis of the torques around the magnetic axis acting on the mode, we argue that it behaves as a kink soliton, which is a solution of the sine-Gordon equation. Such solutions have been studied in the case of the propagation of a fluxon along a Josephson-junction transmission line $^{26-29}$ and we shall follow a similar analysis here.

In order to develop the sine-Gordon equation model, we make the following physically reasonable plausibility assumptions about the nature of the slinky mode: First, we assume that the mode is generated by magnetic reconnection and is an isolated magnetic island of finite length that wraps around the magnetic axis of the torus as shown in Fig. 2. Second, just as is the case in the main magnetic confinement surfaces, we assume that there is a component of the current in the island that flows poloidally around its magnetic surfaces producing magnetic moments along the length of the island. The mode threads its way along the torus passing both the poloidal and toroidal vacuum chamber gaps, and the equation of motion for the slinky mode can be obtained by summing torques ${ }^{6,7,9,20}$ acting on the mode around the magnetic axis. In particular, these torques, which are proportional to the confining magnetic fields, vary sinusoidally ${ }^{20}$ around the mode trajectory due to the toroidal effect.

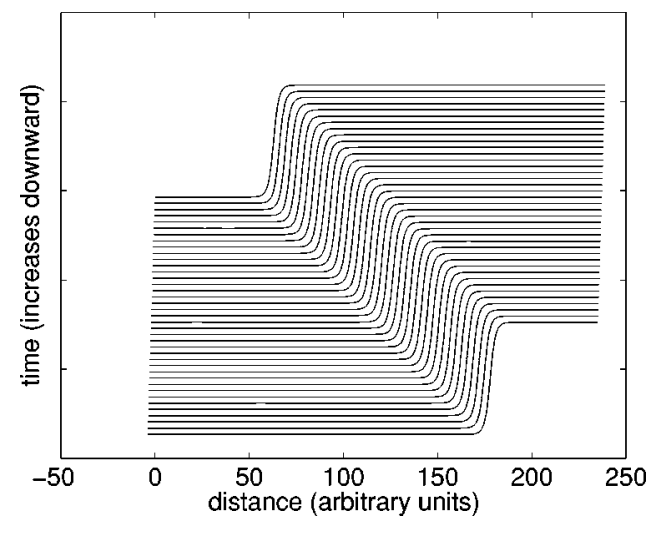

FIG. 3. A kink soliton $\phi(x, t)$ with no driving, damping or gap terms and $v=0.5$.

\section{Derivation of the basic sine-Gordon model}

We further assume that both the toroidal and poloidal magnetic fields at the location of the island vary poloidally and the variation is given by the following expression:

$$
B_{j}=\frac{B_{0 j}}{1+\Lambda_{j}(r) \frac{r}{R} \cos \phi} \cong B_{0 j}\left(1-\Lambda_{j}(r) \frac{r}{R} \cos \phi\right),
$$

where, as shown in Fig. 2, $r$ is the minor radius, $R$ is the major radius of the torus, and $\phi$ is the poloidal angle that can be used to locate the island at each position along its length. $j$ is either $T$ for toroidal or $P$ for poloidal. The terms $\Lambda_{j}(r)$ are less than $1 . \Lambda_{P}$ is negative which ensures that the poloidal field is larger on the outside of the torus.

The magnetic energy of the slinky mode in these fields can be expressed as

$$
W_{\text {mag }}=\mu \cdot \mathbf{B}=\mu_{P} B_{P}+\mu_{T} B_{T} .
$$

A force in the $\phi$ direction results from the $\boldsymbol{\nabla}(\mu \cdot \mathbf{B})$ force acting on the magnetic moments of the island with a resulting torque around the magnetic axis of the torus given by

$$
T=\left(\Lambda_{P} \mu_{P} B_{0 P}+\Lambda_{T} \mu_{T} B_{0 T}\right)\left(\frac{r}{R}\right) \sin \phi=T_{0} \sin \phi .
$$

Thus the torque can be generated by both the toroidal and poloidal fields. Nevertheless, we assume that the slinkymode poloidal cross section is such that it is not located exactly at the toroidal-field reversal layer where the net torque would be very small.

We assume that the island is long enough so that the circulating current around its magnetic surfaces may be considered to be a solenoid; each incremental section of the island is considered to be a circular loop around which current flows. Since the magnetic forces on each loop act so as to produce alignment of the loops, any twisting of the loops will result in a springlike restoring torque which we model in a finite-difference form as

$$
T_{\text {restoring }}=K\left[\phi_{i+1}-2 \phi_{i}+\phi_{i-1}\right],
$$




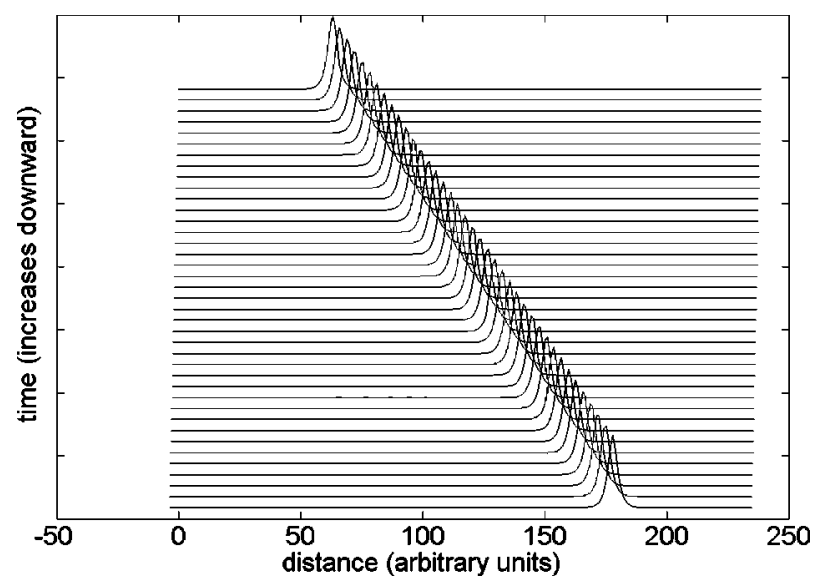

FIG. 4. The derivative of $\phi(x, t)$ with respect to the distance $x$ with $v=0.5$.

where $K$ is the torsional spring constant and $\phi_{i}$ is the angular displacement of the $i$ th loop. Passing to the limit as the separation between the loops tends to zero and summing torques around the magnetic axis, we arrive at the sine-Gordon (SG) equation

$$
M \frac{\partial^{2} \phi}{\partial t^{2}}=K \frac{\partial^{2} \phi}{\partial x^{2}}-T_{0} \sin \phi,
$$

where $M$ is the moment of inertia per unit length of the slinky mode, $T_{0}$ is the torque constant described above, and $x$ is the helical distance along the mode trajectory. In the equation above, if the torque term is zero, we obtain a linear wave equation with phase velocity

$$
v_{\text {phase }}=\sqrt{\frac{K}{M}},
$$

which is, in this formulation, assumed to be the Alfvén velocity.

We now normalize the sine-Gordon equation above by measuring distance in units of $\left(K / T_{0}\right)^{1 / 2}$ and time in units of $\left(M / T_{0}\right)^{1 / 2}$. We obtain

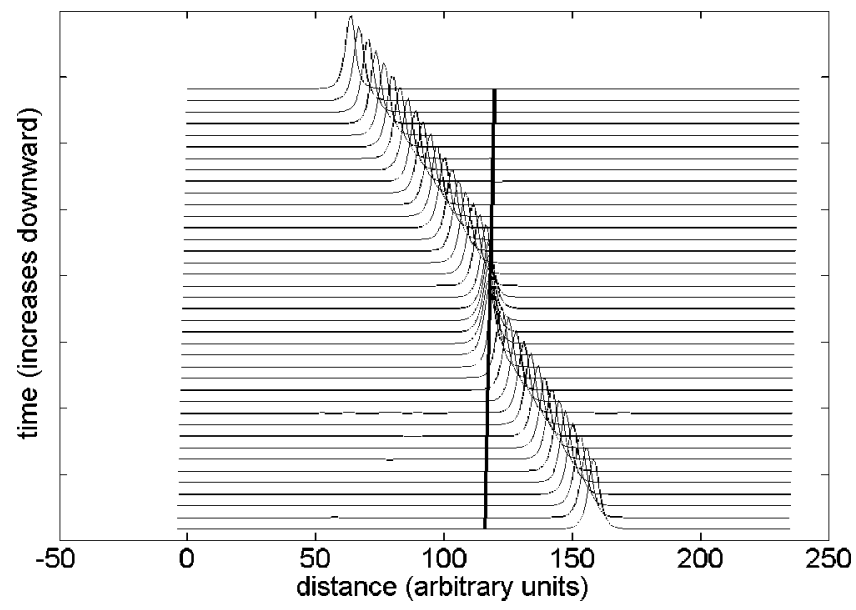

FIG. 5. A plot of a kink with driving and damping terms that passes a gap. Simulation parameters are $t_{\max }=6000, \alpha=0.04, \beta=0, \gamma=-0.02, v=0.5$, and $\sigma=7.075$.

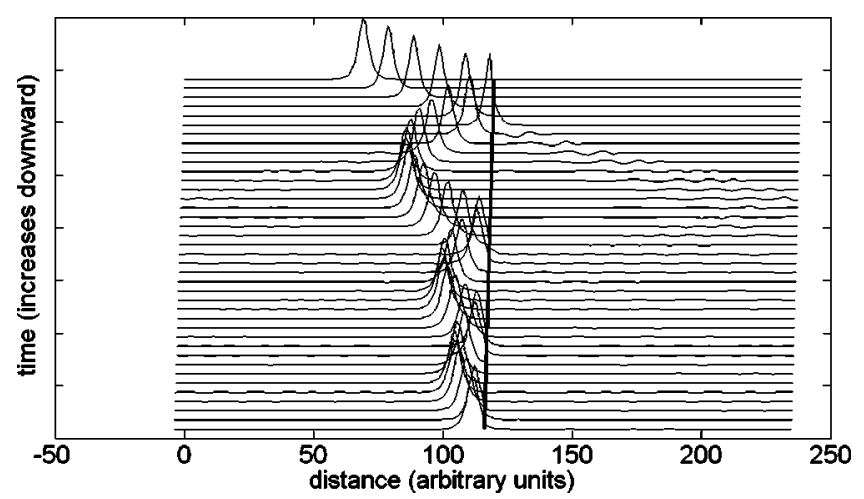

FIG. 6. A plot of a kink with driving and damping terms that cannot pass the gap and locks there. Simulation parameters are $t_{\max }=15000, \alpha=0.01, \beta$ $=0, \gamma=-0.01$, and $\sigma=30$.

$$
\frac{\partial^{2} \phi}{\partial t^{2}}=\frac{\partial^{2} \phi}{\partial x^{2}}-\sin \phi
$$

and note that an exact analytic solution to this equation is a kink soliton ${ }^{30}$ of the form

$$
\phi(x, t)=4 \tan ^{-1}\left[ \pm \exp \left(\frac{x-v t}{\sqrt{1-v^{2}}}\right)\right]
$$

with parameter $v$ denoting the velocity of the soliton, normalized with respect to the Alfvén velocity. This normalized velocity can take any value between -1 and +1 . The factor $v$ also determines the width of the soliton. It is customary to call the "+" solution a kink and the "-" solution an antikink.

Figure 3 shows a succession of plots of $\phi(x, t)$ corresponding to increasing time along the vertical axis. Figure 4 shows a plot of the derivative $\phi(x, t)$ with respect to $x$. We shall use this derivative representation henceforth because this plot shows interaction details more clearly, and also because as the kink passes a magnetic loop sensor, it introduces a current pulse, whose integral with respect to time is similar to what is shown in Fig. 4.

For the kink soliton, the solution $\phi(x, t)$ corresponds to an angular displacement that changes from 0 to $2 \pi$ radians

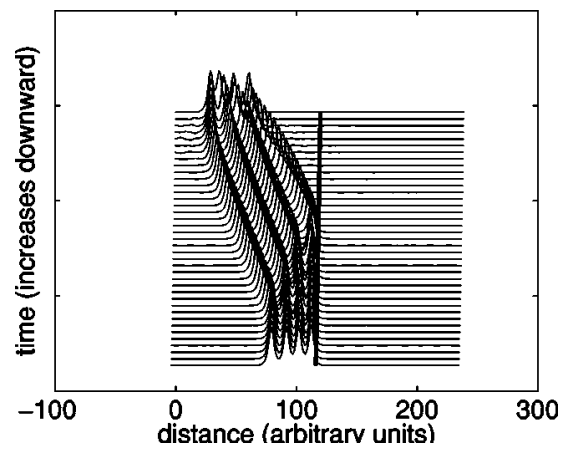

FIG. 7. The trajectories of four kinks launched at separate positions toward the gap. The conditions were set to achieve locking. Simulation parameters are $t_{\max }=10000, \alpha=0.04, \beta=0, \gamma=-0.01, \sigma=30$, and $v=0.5$. 


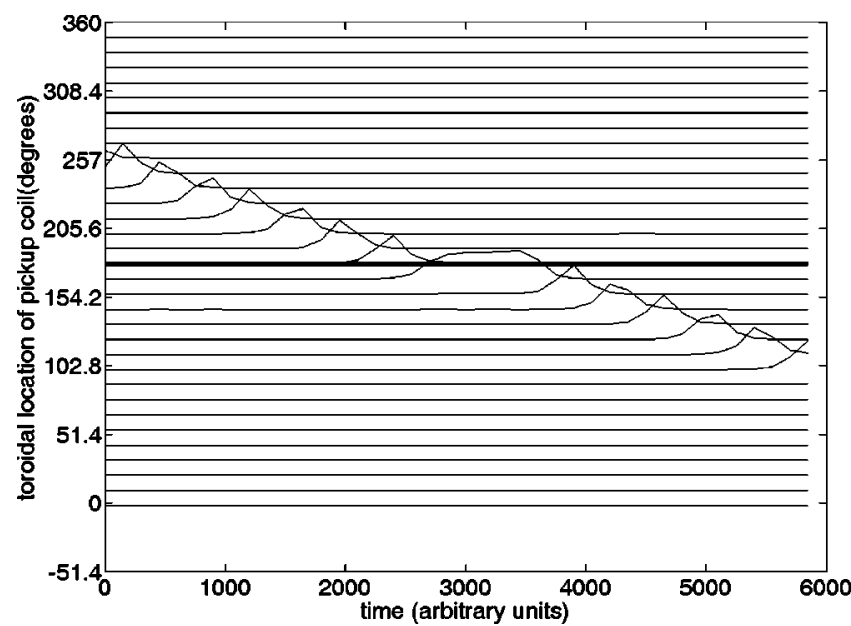

FIG. 8. A time sequence of thirty-two separate sampling points spaced uniformly around the torus. The gap is the solid horizontal line and the vertical axis is toroidal angle.

centered about the point where its argument is zero as shown in Fig. 3. This corresponds to the motion of a set of coupled pendula. ${ }^{30}$ The analog of this trajectory as applied to a torus is as follows. The axial trajectory is the path of the slinky mode as it moves helically around the torus and the center of the trajectory represents the toroidal magnetic axis. Without damping or driving terms, the kink moves with its initial velocity along its trajectory without change. This property is the result of a balance between nonlinear and dispersive effects.

\section{Extended model to include damping, driving, and vacuum chamber gaps}

We now turn our attention to the effects of damping, driving terms, and the vacuum chamber gaps. To study these effects, the sine-Gordon equation is modified as shown below:

$$
\begin{aligned}
M \frac{\partial^{2} \phi}{\partial t^{2}}+D \frac{\partial \phi}{\partial t}-F \frac{\partial^{3} \phi}{\partial t \partial x^{2}}= & K \frac{\partial^{2} \phi}{\partial x^{2}}-T \sin \phi+G \\
& +V_{G} \delta\left(x-x_{0}\right) \sin \phi,
\end{aligned}
$$

where $D$ and $F$ are damping coefficients, $G$ is a driving term, and the $\delta$ function at location $x=x_{0}$ represents the presence of a "gap" in the vacuum chamber whose interaction strength with the slinky mode is $V_{G}$.

More specifically, the effect of the gap can be studied by modeling the interaction of the slinky mode with the gap in the following way. First, during RFP operation, a toroidal image current is induced along the conducting shell. When the current in the shell travels near the gap, continuity of the current is made through an external set of windings that allows the current to flow around the gap. As a result, the current in the conducting shell becomes a poloidal surface current that flows in opposite directions on each side of the gap. The result is a magnetic field in the minor radial direction. Second, due to the same toroidal effects that cause the toroidal magnetic field to vary poloidally, the poloidal surface currents, and hence the radial magnetic field, exhibit a
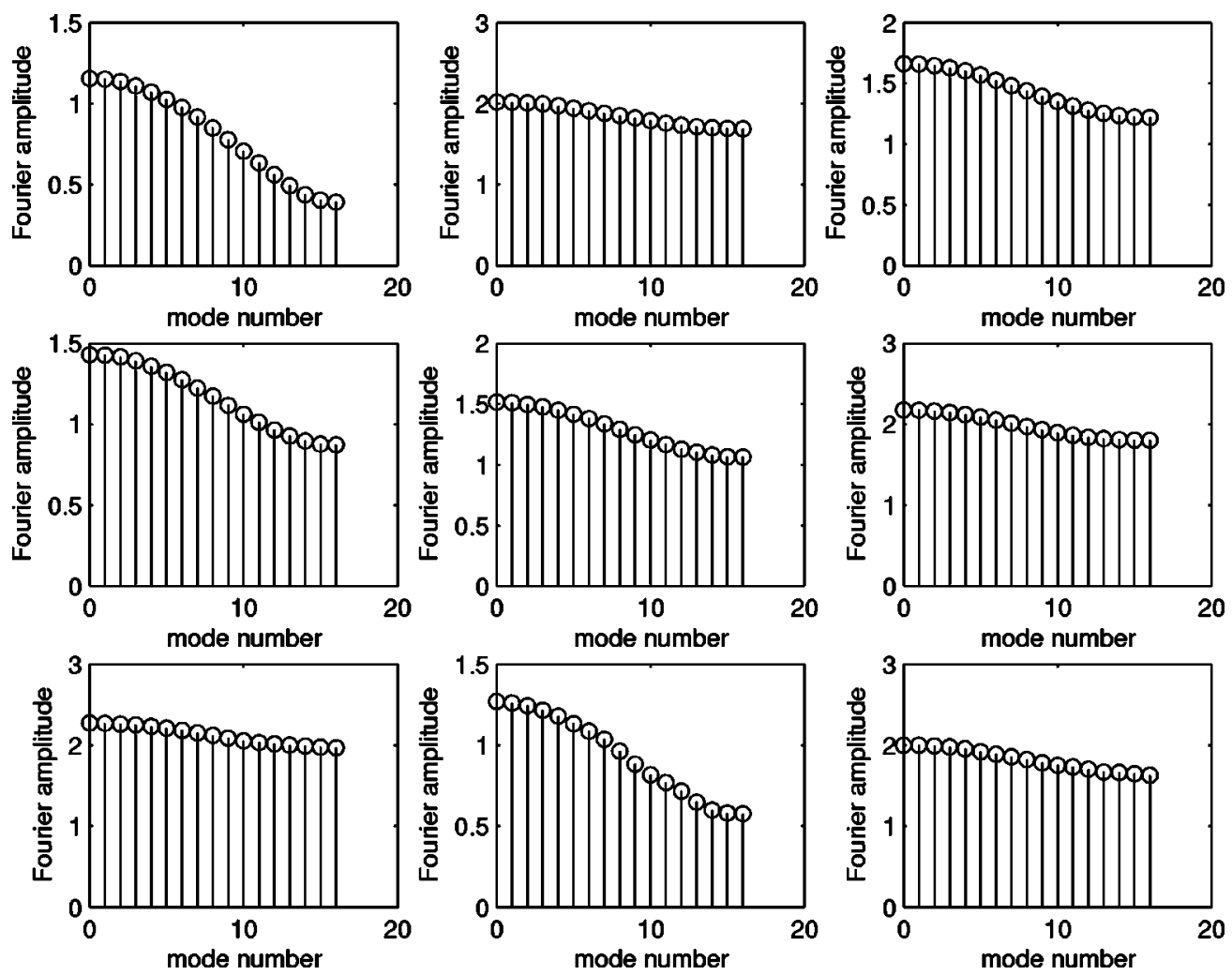

FIG. 9. The fundamental and the first 16 harmonics of the 32 sampled signals for nine separate times. Time increases from left to right and then from top to bottom. 


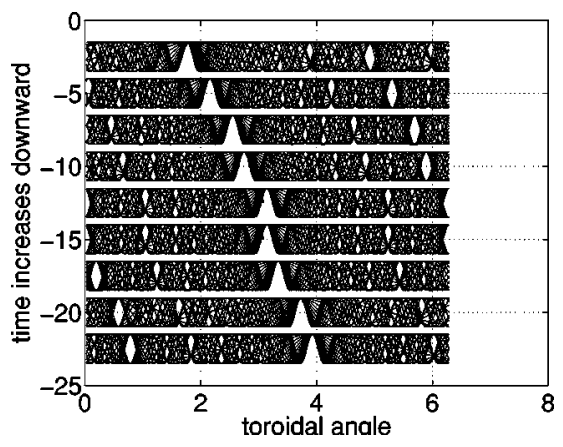

FIG. 10. The overlay of the fundamental and the first 16 normalized harmonics of the kink soliton shown in Fig. 5. The slowing up of the kink soliton as it passes the gap is clearly evident.

similar $\sin \phi$ variation with poloidal angle as does the toroidal magnetic field.

Finally, the radial magnetic field crossed with the toroidal component of the current flowing in the slinky-mode island produces a force in the poloidal direction (and hence a torque about the RFP magnetic axis) which can be in the same or opposite direction as the $\boldsymbol{\nabla}(\mu \cdot \mathbf{B})$ torque depending on the direction of the toroidal current in the slinky mode and/or the direction of the radial magnetic field produced by the surface currents at the gap. Since this torque is localized toroidally to the region at the gap, we model it with the $\delta$ function as shown in the preceding equation. Normalizing the preceding equation we obtain

$$
\begin{aligned}
\frac{\partial^{2} \phi}{\partial t^{2}} & +\alpha \frac{\partial \phi}{\partial t}-\beta \frac{\partial^{3} \phi}{\partial t \partial x^{2}} \\
& =\frac{\partial^{2} \phi}{\partial x^{2}}-\sin \phi+\gamma+\sigma \sin \phi \delta\left(x-x_{0}\right) .
\end{aligned}
$$

The coefficients $\alpha$ and $\beta$ above represent the coefficients of normalized damping terms, the normalized driving term has coefficient $\gamma$ and the normalized strength of the gap-slinky mode interaction is denoted by $\sigma$.

We assume that the damping is produced by plasma turbulence and is proportional to the velocity of the kink. For simplicity, we limit the damping terms to the coefficient $\alpha$. That is, we assume $\beta=0$. We also assume that the driving term is proportional to the plasma current $I_{p}$ and the inter-

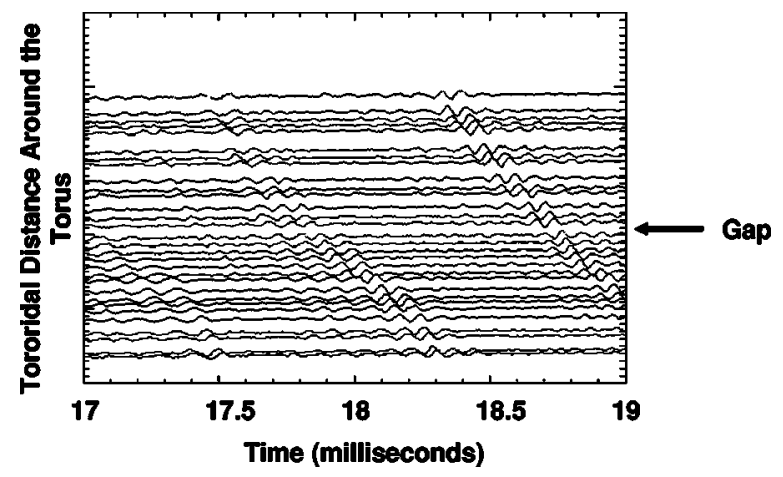

FIG. 11. Thirty-two sampled magnetic pickup signals from the MST experiment showing the slinky mode moving with a nearly constant velocity.

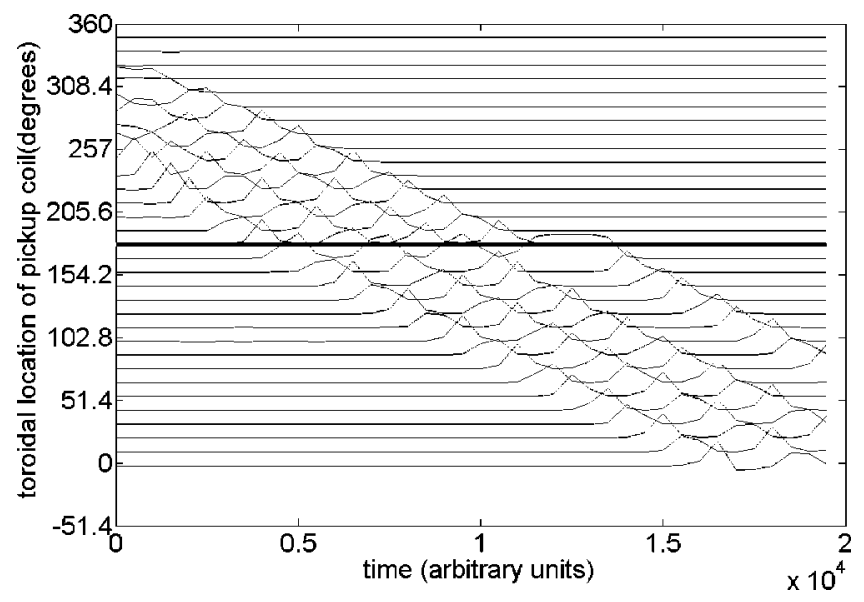

FIG. 12. Thirty-two sampled signals obtained after calculating the trajectories of four kinks passing the gap without locking. Simulation parameters are given by $t_{\max }=20000, \alpha=0.04, \beta=0, \gamma=-0.01, \sigma=1.733$, and $v$ $=0.5$.

action of the kink with the toroidal and poloidal gaps is proportional to the proximity of the kink to the gap(s).

We now turn to the combined effect of the driving and damping terms. The driving term $\gamma$ will accelerate the kink. Its velocity will increase until the deceleration produced by the damping term $\alpha(\partial \phi / \partial t)$ will just balance the acceleration of the driving term. Then the kink will proceed at a steady-state velocity which is generally not equal to the initial velocity that was used to set it in motion.

\section{E. Simulation results}

We solve the modified SG equation using a finitedifference alogrithm. ${ }^{31}$ Figure 5 shows the effect of the driving and damping terms on a kink launched with an initial value of $v=0.5$. In this case, the driving term accelerates the kink until it reaches its "terminal" velocity as it approaches the gap. The gap region is shown as a straight line in the figure.

Next, we examine the effects of the gap. We view the gap as the equivalent of a potential hill or potential well. A positive value for $\sigma$ results in a potential hill for a kink and a potential well for an antikink. Figure 5 also shows the mode trajectory with the gap simulated by a potential hill of

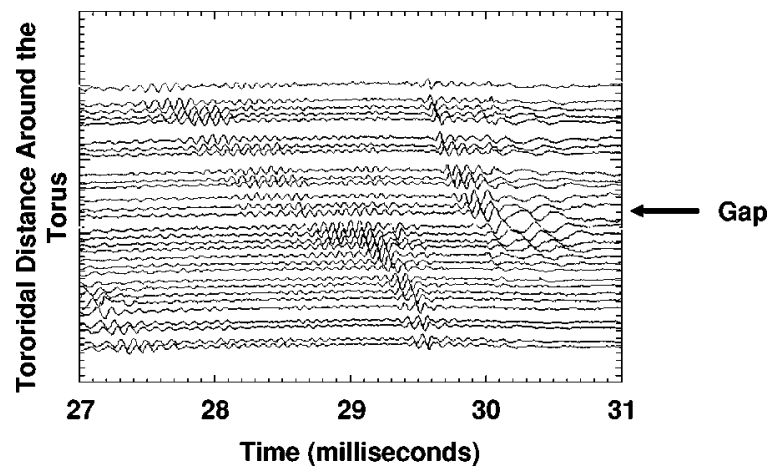

FIG. 13. Thirty-two sampled signals obtained from MST while the slinky mode locks. 


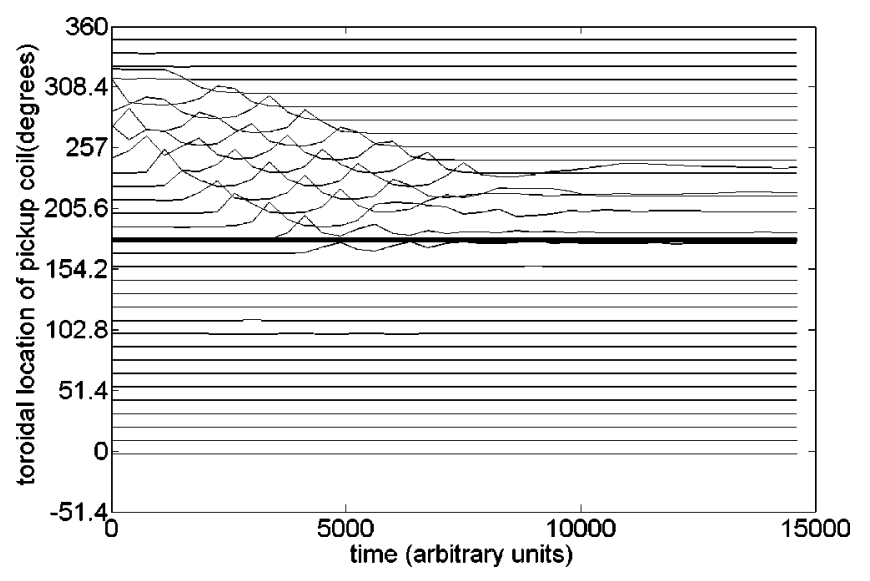

FIG. 14. Thirty-two sampled signals obtained after calculating the trajectories of four kinks that lock just before crossing the gap. Simulation parameters are $t_{\max }=15000, \alpha=0.04, \beta=0, \gamma=-0.01, \sigma=6$, and $v=0.5$.

strength $\sigma$. At low values of $\sigma$, the kink slows down before it reaches the potential hill (gap), and after the kink passes the potential hill, it then accelerates and again eventually reaches its terminal velocity.

Figure 6 shows the results obtained for similar conditions with $\sigma$ being increased. Now, the kink cannot overcome the potential hill and is reflected. The kink turns around after reflection because the driving term eventually overcomes the effects of the reflection. Consequently, the kink returns to the vicinity of the hill where it is reflected again. However, because damping is taking place, the reflected kink moves a shorter distance away from the gap before the kink's velocity again reverses. This type of slinky-mode dynamics has, in fact, been observed experimentally in MST. After a number of these reflections, the kink becomes "locked" to a region directly in front of the hill. For sufficiently high damping, the kink does not reflect and it is locked immediately at the hill.

It is also possible to see these effects when more than one kink is present. Figure 7 shows locking at the gap for a set of four kinks, all launched with the same initial velocity $v=0.5$ but at different positions around the torus. It can be seen that the kinks are preserved as separate entities and no crossing of their trajectories is observed, even during reflections from the gap.

\section{FOURIER DECOMPOSITION OF THE RESULTS}

Experimental measurements of phenomena that occur in toroidal geometry are typically Fourier decomposed in the toroidal and poloidal directions. This has been used to determine which Fourier modes are "dominant" and the analysis of the Fourier modes is often used to predict whether a particular phenomenon will occur. One of the main contentions of this paper is that when such phenomena obey the sineGordon equation, a Fourier analysis is not necessary and can be replaced by the full nonlinear solution. Nonetheless, it is of interest to make a comparison of the theoretical prediction of the sine-Gordon equation with the classical Fourier analysis in the literature.

For the experiment in question, MST, the slinky mode is detected with an array of 32 magnetic field pickup coils spaced uniformly around the torus at the same poloidal angle. Accordingly, in our theoretical model, we also sample the solutions obtained in the preceding section at 32 uniformly spaced points, where we assume that one complete revolution around the torus is represented by the same horizontal dimension in, for example, Figs. 5 and 6. Figure 8 shows the 32 sampled signals as a function of time for the same conditions shown in Fig. 5. Note that in Fig. 8, consistent with experimental data, time is on the horizontal axis and distance around the torus is the vertical axis.

The set of these sampled signals can now be Fourier decomposed in space at various times giving a sequence of "snapshots." Figure 9 shows the spatial Fourier decomposition of the sampled signals of Fig. 8 for nine separate times. Since only 32 sample points are used, only the fundamental and the first 16 harmonics are meaningful. The apparent oscillations observed in the Fourier spectra are due to the fact that the harmonics above the 16 were not obtained from the
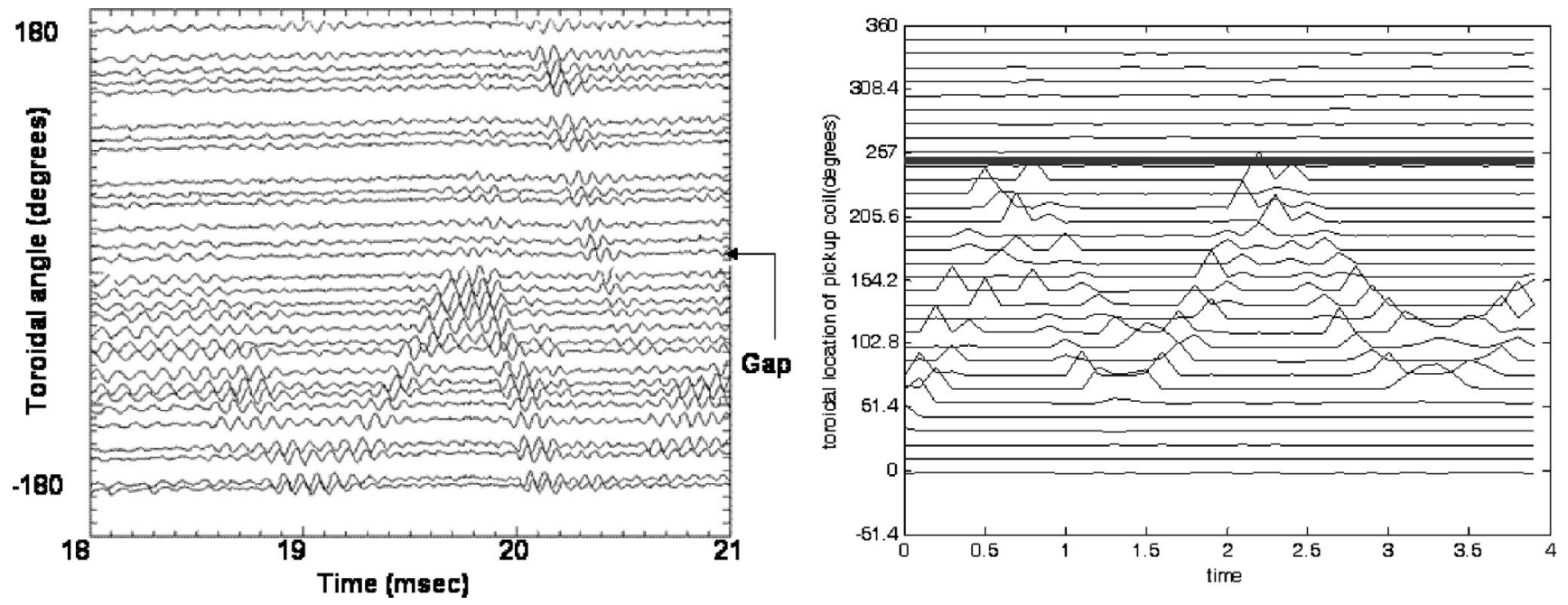

FIG. 15. Experimental vs theoretical comparison of multiple reflections of the slinky mode from the gap followed by reversal of the trajectory due to the driving term. 


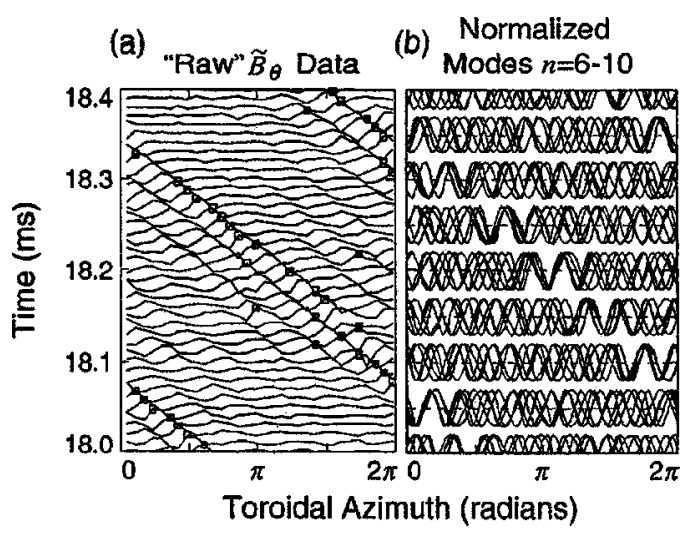

FIG. 16. Overlay of the normalized sixth through the tenth harmonics (b) as taken from the sampled experimental data shown on the left (a). Phase locking is evident.

simulation. If a higher number of harmonics are obtained by additional sampling, it is seen that the spectrum remains constant throughout the simulation.

Using the Fourier coefficients obtained as above, it is now possible to reconstruct the original kink soliton at each instant of time at which the Fourier coefficients were evaluated. It is also possible to examine one or more of the harmonics to determine their relationship and see when and if "phase locking" of specific modes occurs. In order to examine this more easily, we look at the normalized Fourier modes. That is, we assume each mode has unit amplitude but its phase $\varphi_{k}$ is that obtained from the Fourier decomposition. Thus, the $k$ th normalized Fourier-decomposed mode is

$$
\phi_{n o r m, k}=\cos \left[k \frac{2 \pi x}{L}+\varphi_{k}\right]
$$

where $L$ is the distance around the torus.

Figure 10 shows an overlay of the fundamental and the first 16 harmonics at several separate times. Since the modes are each normalized to unity, the representation of the slinky mode is not correct in amplitude, but the phase locking of all of the modes is quite apparent, both before and after the mode interacts with the gap. The calculation is made for the same conditions as shown in Fig. 5.

\section{COMPARISON WITH EXPERIMENTAL RESULTS}

This section provides a comparison of the theoretical work described above with measurements of the slinky mode in the MST experiment. In MST, consistent with the theory in Sec. III, we observe the slinky mode at the locations of the 32 toroidally spaced pickup coils that are placed at a fixed poloidal angle around the MST toroidal conducting shell. We investigate cases where the slinky mode locks and where it does not lock.

Figure 11 shows an experimentally measured slinky mode moving with constant velocity and viewed exactly in the same format as for the theoretical calculations of Fig. 8 . We model the structure of the slinky mode shown in this figure with a chain of multiple kinks, each one rotating about the magnetic axis by $2 \pi$ radians. To represent this, we use, as an example, four kinks passing the gap that are represented as 32 samples in an equivalent way to the experimental results in Fig. 11. These are shown in Fig. 12. Note that in both figures the kinks appear to interact with each other and/or the gap so that their separation as they pass the gap is not constant. As will be shown subsequently, groups of kinks, as in these figures, will show peaks in their Fourier spatial spectra. The mode number(s) where the peak(s) occur will depend on the number of kinks and their relative separation.

A similar comparison can be made in the case of locking. Figure 13 shows the 32 sample experimental measurements of the slinky mode for locking while Fig. 14 gives the results for an equivalent simulation.

One of the more interesting slinky-mode trajectories shows reflection from the gap followed by reversal of the trajectory followed by reversal, etc. Figure 15 displays the experimental results on the left with a corresponding simulation on the right. We conclude from this that the driving term continually acts in the same direction on the slinky

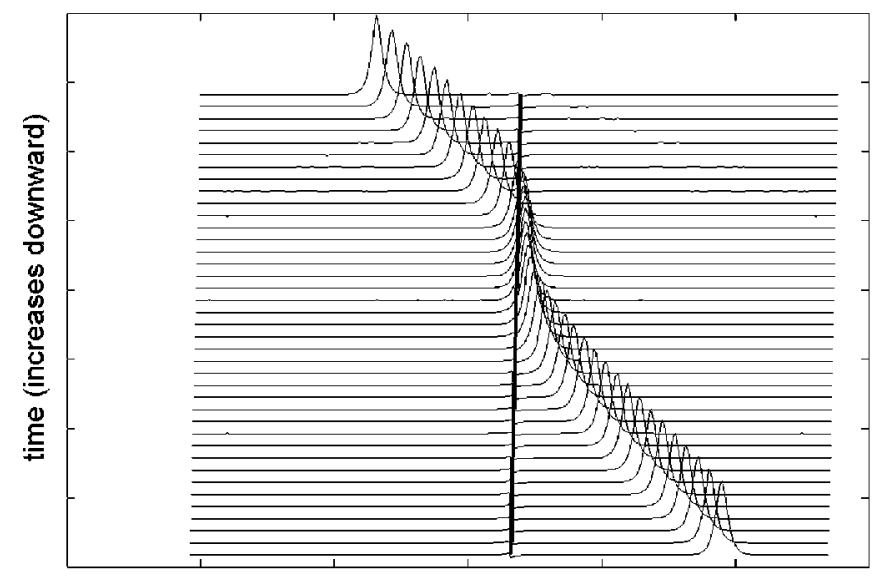

distance around the torus

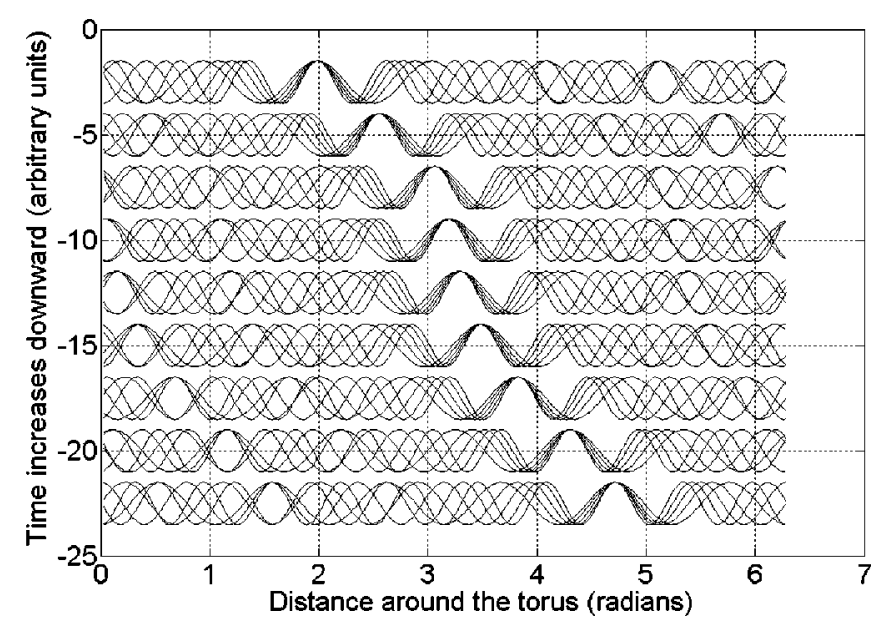

FIG. 17. Overlay of the normalized sixth through the tenth harmonics for the computed kink trajectory shown on the left. Similar phase locking is observed compared with the experimental results shown in Fig. 16. 

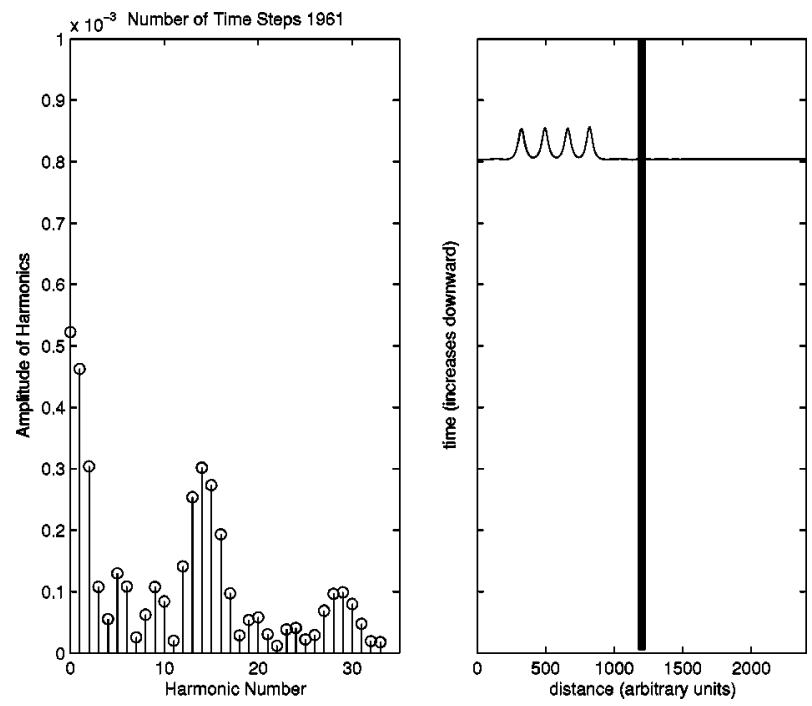

FIG. 18. Spatial Fourier spectrum of four kinks showing a peak at $n=13$. Simulation parameters are $t_{\max }=10000, \alpha=0.04, \beta=0, \sigma=30$, and $v$ $=0.05$.

mode throughout the trajectory and is roughly constant.

We now examine the experimental Fourier decomposition of the slinky mode. ${ }^{3}$ The left side of Fig. 16 shows the experimentally measured slinky mode. The right side of Fig. 16 shows the overlay of the sixth through the tenth normalized modes of the sampled data on the left side of Fig. 16. Phase locking is clearly evident at those locations where the slinky mode appears. It should be mentioned that the experimental Fourier decomposition was made only in the toroidal direction because the slinky mode appears to have only a single poloidal mode number, $m=1$.

The experimental results in Fig. 16 are virtually identical to the results obtained from the solution of the sine-Gordon equation as shown in Fig. 17, including the appearance of phase locking at the points where the kink soliton appears. To make a more direct comparison, Fig. 16 shows the sum of the normalized sixth through tenth harmonics on the right compared with the computed kink trajectory on the left. The resemblance to Fig. 16 is striking.

Finally, it has been reported in the literature that the harmonic spectrum of the slinky mode in MST has a local peak in the spectrum around $n=6 .{ }^{14}$ It is our view that such a peak appears because, in MST, as has been seen in the experimental data in this paper, the slinky mode is composed of a series of kinks. To verify this, Fig. 18 displays the Fourier decomposition of a series of four kinks. The kinks are displayed on the right-hand side of the figure while the Fourier spectrum is displayed on the left-hand side. Note that a peak in the spectrum appears around $n=13$. When the spacing between the kinks is increased and/or the number of kinks is increased, the peak in the spectrum shifts to lower spatial frequencies and vice versa.

\section{CONCLUSIONS}

In this work, it was argued that the use of the sineGordon (SG) equation as a model for the slinky mode in a reversed field pinch device is an effective tool. It is fully nonlinear and does not require the use of Fourier decomposition of the slinky mode. It successfully demonstrates the conditions under which locking of the slinky mode can occur. In addition, we show that phase locking of spatial Fourier modes can occur whenever a localized phenomenon such as the slinky mode appears. Further applications of this technique can be used to explain the change in the experimentally measured width of the slinky mode as the velocity of the mode changes.

Although the SG model we have proposed appears to be much simpler than previous formulations for slinky-mode dynamics, its derivation rests on two well-established facts (1) magnetized cylindrical plasma columns support helical modes and (2) toroidal geometry introduces a periodic poloidal dependence. These two general features lead directly to the SG model. Given the variety of empirical evidence that this model manages to simulate, we believe that the relative simplicity of the SG model should be considered a virtue.

\section{ACKNOWLEDGMENTS}

The authors would like to express their thanks to S.C. Prager and A.F. Almagri and to the reviewer of this manuscript for their encouragement and helpful suggestions.

${ }^{1}$ H. K. Ebraheem, J. L. Shohet, and A. C. Scott, Phys. Rev. Lett. 88, 235003 (2002).

${ }^{2}$ A. F. Almagri, S. Assadi, S. C. Prager, J. S. Sarff, and D. W. Kerst, Phys. Fluids B 4, 4080 (1992).

${ }^{3}$ J. S. Sarff, S. Assadi, A. F. Almagri, M. Cekic, D. J. Den Hartog, G. Fiksel, S. A. Hokin, H. Ji, S. C. Prager, W. Shen, K. L. Sidikman, and M. R. Stoneking, Phys. Fluids B 5, 2540 (1993).

${ }^{4}$ R. Fitzpatrick, S. C. Guo, D. J. Den Hartog, and C. C. Hegna, Phys. Plasmas 6, 3878 (1999).

${ }^{5}$ R. Fitzpatrick, Phys. Plasmas 6, 1168 (1999).

${ }^{6}$ C. C. Hegna, Phys. Plasmas 3, 4646 (1996).

${ }^{7}$ R. Fitzpatrick and E. P. Yu, Phys. Plasmas 7, 3610 (2000).

${ }^{8}$ R. Fitzpatrick and T. C. Hender, Phys. Fluids B 3, 644 (1991).

${ }^{9}$ R. Fitzpatrick, Nucl. Fusion 33, 1049 (1993).

${ }^{10}$ E. Lazzaro and M. F. F. Nave, Phys. Fluids 31, 1623 (1988).

${ }^{11}$ K. Kusano, T. Tamano, and T. Sato, Nucl. Fusion 31, 1923 (1991).

${ }^{12}$ G. A. Navratil, C. Cates, M. E. Maule, D. Maurer, D. Nadle, E. Taylor, Q. Xiao, W. A. Reass, and G. A. Wurden, Phys. Plasmas 5, 1855 (1998).

${ }^{13}$ H. Zohm, A. Kallenbach, H. Bruhns, G. Fussmann, and O. Kluber, Europhys. Lett. 11, 745 (1990).

${ }^{14}$ A. K. Hansen, A. F. Almagri, D. J. Den Hartog, S. C. Prager, and J. S. Sarff, Phys. Plasmas 5, 2942 (1998).

${ }^{15}$ Y. Yagi, S. Sekine, H. Sakakita, H. Koguchi, K. Hayase, Y. Hirano, I. Hirota, S. Kiyama, Y. Maejima, Y. Sato, T. Shimada, and K. Sugisaki, Fusion Eng. Des. 45, 409 (1999).

${ }^{16}$ Y. Yagi, H. Koguchi, A. J. B. Nilsson, T. Bolzonella, P. Zanca, S. Sekine, K. Osakabe, and H. Sakakita, Jpn. J. Appl. Phys., Part 2 38, L780 (1999).

${ }^{17}$ Y. Yagi, H. Koguchi, H. Sakakita, S. Sekine, Y. Maejjima, J. A. B. Nilsson, T. Bolzonella, and P. Zanca, Phys. Plasmas 6, 3824 (1999).

${ }^{18}$ Y. Yagi, H. Sakakita, S. Sekine, H. Koguchi, S. Kiyama, and T. Osakabe, Fusion Eng. Des. 46, 47 (1999)

${ }^{19}$ R. Bartiromo, T. Bolzonella, A. Buffa, G. Chitarin, S. Martini, A. Masiello, S. Ortolani, R. Piovan, P. Sonato, and G. Zollino, Phys. Rev. Lett. 83, 1779 (1999).

${ }^{20}$ A. K. Hansen, Ph.D. thesis, University of Wisconsin-Madison, 2000.

${ }^{21}$ S. Assadi, S. C. Prager, and K. L. Sidikman, Phys. Rev. Lett. 69, 281 (1992).

${ }^{22}$ R. J. La Haye, N. Carlstrom, R. R. Goforth, G. L. Jackson, M. J. Schaffer, T. Tamano, and P. L. Taylor, Phys. Fluids 27, 2576 (1984).

${ }^{23}$ Y. Yagi, H. Koguchi, H. Sakakita, S. Sekine, P. R. Brunsell, and J. A. Malmberg, Phys. Plasmas 8, 1625 (2001).

${ }^{24}$ T. Tamano, W. D. Bard, C. Chu, Y. Kondoh, R. J. LaHaye, P. S. Lee, M. Saito, M. J. Schaffer, and P. L. Taylor, Phys. Rev. Lett. 59, 1444 (1987). 
${ }^{25}$ G. B. Whitham, Linear and Nonlinear Waves (Wiley, New York, 1974), p. 527.

${ }^{26}$ T. A. Fulton and R. C. Dynes, Solid State Commun. 12, 57 (1973).

${ }^{27}$ A. C. Scott, F. Y. F. Chu, and S. A. Reible, J. Appl. Phys. 47, 3272 (1976).

${ }^{28}$ T. A. Fulton and L. N. Dunkleberger, Appl. Phys. Lett. 22, 232 (1973).
${ }^{29}$ P. Guret, IEEE Trans. Magn. MAG-11, 751 (1975).

${ }^{30}$ A. C. Scott, Nonlinear Science: Emergence and Dynamics of Coherent Structures, 2nd ed. (Oxford University Press, New York, 2003), p. 72.

${ }^{31}$ P. S. Lomdahl, O. H. Soerensen, and P. L. Christiansen, Phys. Rev. B 25, 5737 (1982). 